\title{
REVISIÓN DE LA INCORPORACIÓN DE LA ARQUITECTURA ORIENTADA A SERVICIOS EN LAS ORGANIZACIONES.
}

\section{REVIEW OF THE INCORPORATION OF SERVICE-ORIENTED ARCHITECTURE IN ORGANIZATIONS}

\author{
* Msc. Alveiro Alonso Rosado Gomez, Esp. Juan Camilo Jaimes Fernández \\ *Universidad Francisco de Paula Santander Ocaña. \\ Facultad de Ingenierías, Grupo de Investigación de Tecnología y Desarrollo en Ingeniería. \\ Vía Acolsure, Sede el Algodonal - Ocaña, Norte de Santander, Colombia. \\ Tel.: +57 5690088 Ext. 191. \\ E-mail: (jcjaimesf, aarosadog) @ufpso.edu.co.
}

\begin{abstract}
Resumen: Las Tecnologías de la Información se ha transformado en la tecnología de negocios de las empresas modernas, son empleadas para gestión de los procesos y por ende de la información que a través de ellos se maneja, por lo que surge el gran desafío de cómo mejorar la capacidad de los sistemas, en este sentido se hace evidente el papel de la arquitectura orientada a servicios (SOA) la cual permite la integración se servicios para que varias aplicaciones puedan acceder desde un solo lugar mejorando significativamente la gestión empresarial desde diferentes contextos como el empresarial, el médico, el militar y el internet de las cosas.
\end{abstract}

Palabras clave: Integración de procesos, integración de tecnología, servicios reutilizables, servicios Web, SOA.

Abstract: Information Technology has been transformed into the business technology of companies, they have been used for the management of the processes and for the information that is handled through them, so the great challenge arises of how to improve the capacity of the systems, in this sense it becomes evident the role of service oriented architecture (SOA) which allows the integration of services so that several applications can be accessed from a single place improving business management from different contexts such as business, the doctor, the military and the internet of things.

Keywords: Process integration, technology integration, reusable services, Web services, SOA. 


\section{INTRODUCCIÓN}

En los orígenes de las Tecnologías de la información (TI) todo el énfasis estuvo puesto en desarrollar sistemas que automatizaran tareas que se hacían manualmente con el fin de agilizarlas (Bazán, Fernández, Molinari, Pérez, \& Banchoff, 2017), actualmente el hecho de que muchas empresas se encuentran a la vanguardia en este mundo cada vez más competitivo se debe a que han acogido las TI como factor primordial en su quehacer diario. Las TI hacen referencia a la utilización de medios informáticos como equipos de telecomunicaciones para almacenar, procesar y difundir todo tipo de información o datos en las distintas unidades que conforman cualquier organización (De Jesús, 2013).

A medida que las economías experimentan un cambio estructural significativo, las organizaciones se ven obligadas competitivamente a aprovechar la computación en la nube para expandir o contraer su huella informática en función de las demandas variables de recursos informáticos. (SHENG \& RANJAN, 2017). Las TI, se ha transformado en la tecnología de negocios de las empresas modernas, son empleadas para gestión de los procesos y por ende de la información que a través de ellos se maneja (Nagarajan, 2013); las instituciones de educación superior son un ejemplo de ello, muchos de los procesos se apoyan en las TI para una ágil gestión tanto los actuales como de aquellos que surgen debido a nuevos requerimientos $\mathrm{y}$ al crecimiento institucional, por lo que surge el gran desafío de cómo mejorar la capacidad de los sistemas para agregar nuevas interfaces, combinar múltiples fuentes de datos en una sola, interactuar con dispositivos móviles y reemplazar viejas aplicaciones con nuevas (GARCIA, 2014). El éxito de las empresas depende de su capacidad de gobernar los recursos incluyendo los de las TI de forma efectiva; es necesario además establecer y aplicar estrategias de gestión alineadas con los objetivos (Tarouco \& Reis, 2010).

Además el crecimiento constante de delitos informáticos como espionaje, robo de propiedad intelectual, fraude, entre otros, implica que las organizaciones tomen medidas que además les permitan manejar de forma segura la información. (Santos \& Flórez, 2012).

La implementación de la Arquitectura orientada a servicios (Service Oriented Architecture-SOA) constituye una estrategia idónea pues permite estructurar los procesos del negocio y la tecnología

Universidad de Pamplona para responder de forma ágil y flexible a los requerimientos a la par con los objetivos planteados (CAR, 2008).

(SOA) ha sido introducida para fomentar una interacción dinámica y de bajo acoplamiento entre servicios ofrecidos por diferentes proveedores, permitiendo el desarrollo de sistemas distribuidos altamente escalables, sus principales objetivos consisten en soportar la interoperabilidad de servicios proveniente de diferentes proveedores y facilitar modificaciones que permitan al sistema evolucionar. (Ibáñez, 2015)

Esta investigación expone un panorama de los entornos organizacionales en donde se viene aplicando SOA; para lograrlo se parte de los elementos conceptuales que la constituyen, posteriormente se profundiza en la incorporación de la arquitectura como la solución adecuada a una necesidad de integración de servicios que requería la TI en la organización.

\section{MÉTODO}

Para la localización de documentos bibliográficos se implementaron búsquedas en diversas fuentes de información; específicamente en bases de datos digitales como IEEE Computer Society, ScienceDirect Scopus y ACM Digital Library. También se indago en revistas científicas como Redalyc, en repositorio institucionales de universidades nacionales y extranjeras, los parámetros de búsqueda establecidos tienen como punto de partida SOA y su aplicación en diferentes contextos, la información referente a la aplicación de SOA se tomó de un rango de fecha no mayor a 7 años de antigüedad a excepción de aquellas definiciones específicas.

\section{ARQUITECTURA ORIENTADA A SERVICIOS (SOA)}

SOA, es un paradigma tecnológico ampliamente difundido, que a través de los años ha apoyado a múltiples organizaciones en el logro de objetivos estratégicos cada vez más ambiciosos (Oviedo, 2016). SOA, está formada por componentes disponibles a través de interfaces genéricas y protocolos estandarizados y preferentemente libres de licencias (servicios), diseñados con el menor nivel de dependencia posible con los sistemas de información que los consume y de la parte técnica 
del desarrollo, estimulando su reutilización y aprovechamiento de las funcionalidades ya existentes (Serman, 2010). Se trata esencialmente de un conjunto de servicios cohesivos, donde cada uno es relativamente económico de construir o reemplazar si es necesario. Al ser independientes, el poder unirlos permite a SOA adaptarse a los cambios de formas más sencilla que las arquitecturas tradicionales (Gonzáles. 2010).

SOA es un patrón arquitectónico en el diseño de software de computadora en el que los componentes de aplicaciones proporcionan servicios a otros componentes a través del protocolo de comunicaciones. (Dutta, Atora, \& Devi, 2017)

Concretamente SOA es un estilo arquitectural para construir soluciones empresariales basadas en servicios (Romero, 2012). Esto coincide con lo expuesto por Ledgard \& Espinoza (2010), quienes mencionan que SOA proporciona una metodología y un marco de trabajo basado en servicios. Además, está orientada y alineada con el negocio, por lo que normalmente se habla de SOA como un modelo de arquitectura tanto de TI como corporativo.

SOA separa las funciones en unidades o servicios distintos, a los que los desarrolladores hacen accesibles a través de una red para permitir que los usuarios los combinen y reutilicen en la producción de aplicaciones. (Chanda, Sengupta, Kanjilal, \& Bhattacharya, 2013). Se encuentra dentro de los principales estilos de arquitecturas de software conocidos hasta el momento. El principal aporte de la arquitectura SOA es la abstracción de los procesos, por la que los procesos de negocio se externalizan de las aplicaciones o soluciones y se exponen directamente al negocio. Esto permite que la ejecución, gestión, monitorización y modificación de dichos procesos puedan ser manejadas directamente a nivel de negocio y de forma versátil, en vez de estar embebidos en las aplicaciones (Alfonso \& Enciso, 2010).

Hoy en día las tecnologías inalámbricas han adoptado en el transcurso del tiempo una manera más sencilla y cómoda de utilizar toda clase de dispositivos, con el fin de mejorar los procesos y/ó las aplicaciones en general. (Durán \& Iturriago, 2012).

SOA es un paradigma arquitectónico que ha ganado una atención significativa dentro de la TI y las comunidades empresariales. (IEEE Computer

Universidad de Pamplona
Society, 2017), ofrece la oportunidad real de conseguir un salto hacia delante en agilidad y eficiencia y situar a las TI en un nuevo nivel. Esto exige un cambio de una forma de pensar basada en las aplicaciones a una perspectiva que abarque a toda la empresa con el objetivo de controlar la forma en que se cumplen con los flujos de trabajo y la forma en la que se desarrollan, implementan y gestionan los servicios durante todo su ciclo de vida para así cumplir los objetivos de negocios de la empresa. Para lograr dichos objetivos es buena práctica que los servicios que la componen estén diseñados siguiendo elementos propuestos por las mejores prácticas como los presentados en la figura 1 (Granados, 2014).

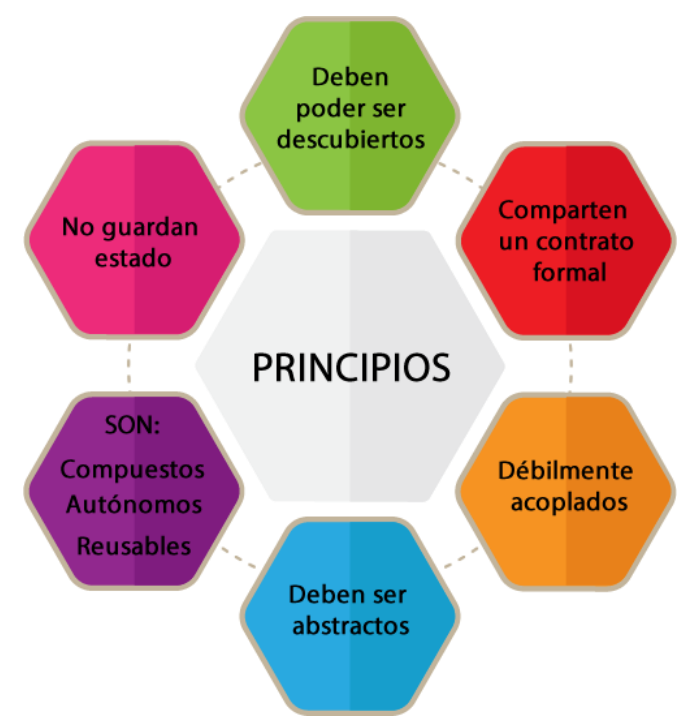

Fig. 1. Principios de SOA

Fuete. Adaptado de (Granados, 2014)

A continuación, se describen cada uno de los principios que se ilustran en la figura 1:

Los servicios son reusables: Los servicios tienen y expresan una lógica agnóstica con lo que pueden ser utilizados por múltiples servicios y/o sistemas.

Los servicios comparten un contrato formal: Un contrato de servicio es la especificación del servicio, en el cual se debe mencionar: Cómo debe ser consumido, su ubicación, reglas y características de las operaciones que ofrece.

Los servicios están débilmente acoplados: Los servicios deben idealmente ser capaces de adaptarse a cambios en sus dependencias en el menor tiempo posible, a bajo costo y de manera transparente. 
Los servicios son compuestos: En ocasiones en un servicio puede estar compuesto por otros, de modo estos conjuntos de servicios pueden encargarse de resolver una tarea más compleja

Los servicios son autónomos: Se debe procurar que los servicios tengan control de sí mismos para realizar sus tareas sin tener dependencias de factores o actores externos a su entorno.

Los servicios deben ser abstractos: Cada petición al servicio debe ejecutarse de forma independiente a las peticiones previas, no debe guardar información de sesión de quién lo haya ejecutado.

Los servicios no guardan estado: Este principio está muy ligado al contrato de los servicios, debe existir un mecanismo de inventario de servicios en los que se especifique su funcionalidad y cómo poder acceder a ellos.

Los servicios deben poder ser descubiertos: El propósito de un servicio es realizar sus tareas sin importar cómo opera internamente, simplemente debe arrojar los resultados obtenidos, de esta forma parecerán como una caja negra, dando importancia al ¿qué? y no al ¿cómo?

\subsection{Ventajas de la Arquitectura orientada a servicios}

Según Alfonso \& Enciso (2010), las principales ventajas que provee la arquitectura orientada a servicios se podrían resumir así:

1. Ayuda a mejorar la agilidad y flexibilidad de las organizaciones.

2. Facilita trabajar con tercerización.

3. Permite la simplificación del desarrollo de soluciones mediante la utilización de estándares de la industria y capacidades comunes de industrialización.

4. Facilita abordar modelos de negocios basados en colaboración con otros entes (socios, proveedores). 5. Ayuda a aislar mejor a los sistemas frente a los cambios generados por otras partes de la organización.

6. Promueve la integración de tecnologías diferentes.

7. Alinea y acerca las áreas de tecnología y negocio.

Las dos ventajas más destacables SOA según González (2011), son la reutilización del software que aporta beneficios sobre la calidad, los costos y facilidad para el despliegue y la independencia de

Universidad de Pamplona

I. I. D. T. A. la plataforma tecnológica y las aplicaciones de la infraestructura.

La educción de requisitos de software es una actividad propia de la primera fase del ciclo de vida del software, en ésta, se utilizan diferentes diagramas que ayudan al analista a efectuar el proceso de identificación y validación de requisitos. (Parra \& Herrera, 2012).

Según Joachim, Beimborn, \& Weitzel (2012), no todas las empresas que han introducido SOA logran los beneficios que se esperan como el aumento de la flexibilidad o la reutilización, solo una de cada cinco y esto se debe principalmente a la falta de gobierno. Lo anterior se reafirma con lo manifestado por INMETRICS (2017) quien afirma que la gobernanza de TI se asocia con la estructuración de un conjunto de directrices, responsabilidades, competencias y habilidades, cuyo objetivo es controlar con eficacia los procesos, garantizar la seguridad de la información y optimizar la aplicación de los recursos, además de dar apoyo a la toma de decisiones.

La seguridad en sistemas informáticos es un tópico de alta importancia hoy en día, debido a que los sistemas de información están interconectados con otros sistemas a través de internet o redes propietarias. (Luna, Lezcano \& Goméz, 2013).

\subsection{Gobierno de SOA}

La gobernabilidad de SOA es una ampliación de la gobernabilidad de TI (Yashar, 2009); dado que el diseño de SOA tiene como objetivos ser robusta, escalable, integra y reutilizable.

El gobierno SOA es el proceso de asegurar que todos los intereses de los participantes en la arquitectura son tenidos en cuenta en la planeación, diseño y ejecución de la SOA de una organización. En este sentido, se hace referencia a la organización, procesos, procedimientos, políticas y métricas requeridas para administrarla exitosamente (Cruz \& Correal, 2011). Se busca establecer las políticas y lineamientos que deben regir esta actividad a través del ciclo de vida de los servicios de negocio y tecnológicos en una organización (Londoño, 2014).

Escobar, Aguilera, \& Parra (2016), establecen que el diseño de una arquitectura orientada a servicios es identificar funcionalidades en un ámbito bien definido y hacerlo accesible de forma uniforme y 
completa, pero independiente a donde se use. Además, dentro de SOA los servicios incorporan reglas de negocios, información y operaciones. Antes de comenzar a crear estos servicios, es necesario contestar las siguientes 2 preguntas: (1) ¿Qué servicios se requieren? y (2) ¿Qué servicios se deben desarrollar?; para dar respuesta a la primera pregunta es necesario tener en cuenta que la mejor manera de detectar servicios es a partir de la modelación del proceso de negocio. Mientras que en la segunda pregunta hay que determinar para cada servicio detectado, si debe ser desarrollado desde cero o si es posible proveer su funcionalidad a otro servicio o actividad.

Paso 1: Identificación de servicios La idea de un servicio es identificar un ámbito bien definido de funcionalidad $y$ hacerlo accesible de forma uniforme y completa, independiente de dónde se use.

Paso 2: Modelado de servicios Los servicios para mantener su independencia, pueden encapsular su lógica dentro de una tarea, subproceso o proceso y establecer relaciones con aquellos que quieren usarlos. Este paso es el siguiente escalón en el perfeccionamiento del modelado del proceso y se realiza en base a la descripción del servicio (paso anterior).

\subsection{Aplicaciones de la arquitectura SOA}

En muchos casos la infraestructura tecnológica en las diversas empresas está soportada por aplicaciones web que hoy en día pueden considerarse obsoletas y que deben ser rediseñadas sobre la base de arquitecturas software más flexibles y que puedan responder a este continuo cambio de requisitos (Sánchez, Clemente, Prieto, \& Rodríguez, 2017).

Es común encontrar en las empresas diversos sistemas TI que soportan los procesos de negocio y que no se comunican entre sí, SOA, apunta a cubrir las necesidades de las empresas en lograr la interoperabilidad de sus tecnologías, permitiendo eliminar procesos manuales y horas hombre dedicadas a traspasar información de un sistema a otro (Carvajal, 2014).

Las organizaciones actualmente para ser competitivas requieren de procesos de negocio automatizados y flexibles para responder rápidamente a los cambios emergentes del entorno. Esta demanda de procesos de negocio flexibles exige enfoques sistemáticos para facilitar la implementación de líneas de procesos de negocio que cubran eficaz y eficientemente las necesidades de las organizaciones y sus clientes (Cardona, 2014).

Organizaciones a nivel nacional e internacional, como Nortel e IBM implementaron la arquitectura SOA para conseguir más eficiencia en las comunicaciones entre los clientes, empleados $\mathrm{y}$ colaboradores a través de nuevas vías sin que se afectara el servicio la seguridad o las inversiones realizadas con anterioridad en tecnología (El Economista América, 2007)

El Poder Judicial de Chile en su afán de agilizar los procesos, ofrece a sus usuarios (funcionarios públicos y abogados) varios portales en donde se puede consultar las diferentes causas y trámites que se llevan a cabo. Estos portales se encontraban publicados en la página oficial de la institución, pero funcionaban de manera independiente por lo tanto sus datos y servicios no estaban integrados y existía duplicidad, por lo que se diseñó e implementó una arquitectura de software orientada a servicios, que permitió dar solución a los problemas de escalabilidad y duplicidad de datos de los sistemas de la institución, permitiendo el manejo integral de la información y unificando los diferentes sistemas que actualmente están en producción (Ochoa \& Perovich, 2017).

La arquitectura SOA constituye la base que garantiza la agilidad del negocio, un prerrequisito fundamental para alcanzar el éxito en el actual mercado mundial, siempre tan competitivo. Esta agilidad es la capacidad de añadir, modificar y optimizar fácilmente los procesos de negocio mediante el aprovechamiento de las sinergias de servicios o procesos. Este aprovechamiento tiene el fin de crear una nueva gama de capacidades o productos, mediante la combinación de algunos elementos de los procesos de negocio actuales, y dando soporte así a nuevos segmentos de clientes, canales o mercados (CAR, 2008).

Los desarrolladores a menudo crean interfaces de servicio que perpetúan los métodos de acceso a datos deficientes, que niega los beneficios de crear servicios que comparten datos. Para dar aplicaciones orientadas al servicio Se debe abordar una base sólida, la calidad de los datos y los problemas de interoperabilidad. (Mohamad, 2007)

La aplicación de un modelo de referencia para lograr una arquitectura completa, equivale a pasar 
de una etapa de análisis a una de diseño en analogía con las etapas del ciclo de vida del software. Implica dar un paso más en el nivel de detalle y comenzar a buscar metodologías para aplicar sobre los conceptos analizados. Una arquitectura concreta se desarrolla en un contexto predefinido donde se fijan protocolos, perfiles, especificaciones y estándares. La plataforma SOA combina estos elementos a los efectos de generar un producto operativo (Bazán, 2009).

\subsubsection{Integración de SOA con Internet de las Cosas (IOT)}

El concepto de Internet de las cosas (IoT) prevé la integración de objetos heterogéneos con capacidades de comunicación por cable e inalámbricas para formar una red ubicua. La última década ha visto la prevalencia de este concepto en entornos domésticos y de oficina no críticos, pero solo hasta los últimos años hemos comenzado a ver este concepto expandirse al dominio industrial. (Raza, Lomax, Ghafir, Kharel, \& Whiteside, 2017).

La visión original de la IoT incluye a un ecosistema global híper conectado en el cual las "cosas" se comunican con otras "cosas", ya sea por la necesidad de entregar servicios altamente diversificados al usuario. Tales comunicaciones deben ser independientes del creador de un determinado fragmento de infraestructura. En la realidad, sin embargo, cada vendedor posee su propia solución de IoT que es incompatible con otras soluciones, generando silos IoT locales (Fortino, Ganzha, Palau, \& Paprzycki, 2016).

Tradicionalmente, los sistemas de una empresa que controla procesos físicos (como la fabricación, la ejecución de logística, la supervisión de energía, la automatización de edificio, etc.) usan normas diferentes para datos y comunicación que para sus sistemas de gestión. A medida que avanzamos hacia el "Internet de las cosas", millones de dispositivos estarán interconectados y consumiendo información disponible en la red, estos dispositivos necesitan interoperar, el enfoque orientado al servicio parece ser una solución prometedora, es decir, cada dispositivo debe ofrecer su funcionalidad como estándar de servicios, mientras que en paralelo es posible descubrir e invocar nueva funcionalidad de otros servicios a pedido (Spiess, y otros, 2016).

El mercado financiero, económico y administrativo se considera un entorno volátil debido a que las tecnologías evolucionan rápidamente y la información se mueve más rápido que en años anteriores. (Hernández, Leal, Nieto, Jimeno, \& Muñoz, 2017).

\subsubsection{SOA orientado a servicios web}

Un servicio web es una aplicación de software autodescriptiva que puede invocarse en la Web utilizando un conjunto de estándares. (Malik, Medjahed, \& Rezgui, 2016).La Arquitectura SOA, establece un marco de diseño para la integración de aplicaciones independientes de manera que desde la red pueda accederse a sus funcionalidades, las cuales se ofrecen como servicios. La forma más habitual de implementarla es mediante Servicios Web, una tecnología basada en estándares e independiente de la plataforma, con la que SOA puede descomponer aplicaciones monolíticas en un conjunto de servicios e implementar esta funcionalidad en forma modular (Montejano, Testa, García, \& Bast 2012). La combinación de servicios web es una tarea importnate que se realiza en diferentes fases del ciclo de vida de al arquititectura orientada a servicios. (Zavvar, Garavand, \& Sabbagh, 2017). Esta arquitectura ha permitido habilitar el entorno distribuido para que la gran mayoría de las aplicaciones basadas en componentes puedan operar entre sí, brindando heterogeneidad al famoso mundo de las aplicaciones distribuidas, da una visión de que el software debe ser entregado como un servicio, orientando al mercado del software hacia un entorno más competitivo con soporte para los negocios. Esto a su vez permite de forma dinámica la creación e implantación de nuevos servicios basados en otros ya existentes (Santos, Rico, \& Rincón, 2009).

\subsubsection{SOA en la industria médica}

Tradicionalmente, el desarrollo de aplicaciones se ha orientado al acceso directo del usuario a través de interfaces gráficas y este enfoque se centra en los flujos del usuario y las funcionalidades esperadas por él. Todos estos sistemas funcionan de manera aislada, debido a la falta de estandarización, la información se almacena en pequeños sistemas incompatibles entre sí, formando "Islas de Información", eliminar las islas o silos de información y cómo realizar la interoperabilidad de forma efectiva son problemas desafiantes en la industria médica. Para promover la interoperabilidad y la manejabilidad, SOA ha sido utilizada por varios años, ya que su naturaleza de bajo acoplamiento permite la 
integración de sistemas legados y puede fácilmente acomodar necesidades en constante evolución, por ser una plataforma extremadamente adaptable, SOA se ha utilizado en diferentes ámbitos, siempre con el propósito de promover la interoperabilidad de software heterogéneo. En el área de la salud no es diferente (Pereira, Nogueira, \& Aparecida, 2016).

De las mayores preocupaciones en clínicas y hospitales se relaciona con el uso y gestión de la información. Los Sistemas de Información que se utilizan en el área de la salud no tienen diferencias sustanciales en comparación a los utilizados en otras industrias, sin embargo, su implementación es tremendamente compleja. Numerosas técnicas han sido propuestas para mejorar la comunicación. (Gélvez, 2012). El origen de esta complejidad radica en la cantidad de sistemas específicos que conforman la solución integral, por este motivo, resulta indispensable diseñar una estrategia que permita gestionar la incorporación de un Sistema de Historia Clínica Electrónica (Etchart, 2015).

SOA permite el intercambio de información de forma segura y confiable a un costo razonable. Es una arquitectura escalable que está pensada para sistemas grandes que deberán evolucionar en el tiempo adquiriendo nuevas funcionalidades y que requerirán de mucho trabajo para completarse. Por eso esta orientación es la ideal para construir un Sistema HCE (Sistema de Historia Clínica Electrónica), los diversos análisis muestran que una arquitectura basada en servicio es perfectamente factible en la industria de la salud. De hecho, empresas como Oracle ofrecen sistemas de integración basada en estos métodos y son ampliamente apoyados en diversos sitios web (Etchart, 2015).

\subsubsection{SOA en la arquitectura empresarial}

Una empresa es una entidad compleja compuesta de personas, procesos y tecnología, que producen productos o servicios, Cualquier organización puede ser estructurada de acuerdo a tres nieles jerárquicos: Estrategia, procesos y sistemas de información; la alineación entre los modelos de negocio y los modelos de gestión tecnológica, difícilmente se logra, si existe una brecha amplia entre las dos perspectivas, es necesario que las áreas de TI desarrollen una visión más abierta y estrechamente conectada con el negocio, así como una nueva alternativa de pensamiento sobre la orientación a servicios de los componentes tecnológicos que provee. La adopción de un modelo de arquitectura orientado a servicios proporciona los mecanismos que permiten definir contratos de prestación de servicios que aseguren que la capa de negocios de una organización se encuentre alineada con la capa de TI (Arango, Londoño, \& Zapata, 2010).

Los sistemas médicos modernos son intensivos en el uso de la tecnología de distribución en los servicios médicos. La comunicación entre profesionales y pacientes está habilitada para intercambiar información del paciente y para el monitoreo en línea de las condiciones de salud. (García, Herrasti, Jouvray, \& Armentia, 2017). La arquitectura de software de las corporaciones debe ser: simple (para que todas las partes interesadas puedan entenderla y utilizarla); flexible (para que pueda acomodar en tiempo las dinámicas modificaciones requeridas por el entorno de negocios); generadora de reutilización (sobre todo de los bloques de software); y ser capaz de desvincular las características del negocio de las tecnologías utilizadas para su ejecución (Sordi, Marinho, \& Nagy, 2006).

La mayoría de las organizaciones de atención médica tienen una gran cartera de sistemas con procesamiento redundante y datos. SOA permite que las capacidades del sistema sean seleccionadas y empaquetadas como servicios que están mejor enfocados y disponibles en toda la organización. Las organizaciones pueden cambiar sus esfuerzos de mantener una estrategia compleja de interfaz de datos para crear aplicaciones orientadas a servicios que admitan la interoperabilidad mientras se alinean más estrechamente con los procesos de atención médica (Juneca, Dournaee, Natoli , \& Birkel , 2008).

\subsubsection{SOA en el contexto militar}

El requisito de permitir una guerra centrada en la red mediante el alojamiento de capacidades habilitadas para la red, promovió el uso de SOA dentro de las redes militares. La respuesta inicial de las comunidades académicas e industriales fue utilizar SOA empresarial estándar. Las soluciones desarrolladas se ajustaron bien al dominio estratégico, donde las restricciones de nodo y red eran mínimas. Sin embargo, la experiencia adquirida en los campos de batalla de la última década, ha demostrado que el dominio táctico impone un conjunto de limitaciones únicas, que hacen que tales soluciones sean ineficientes para la ventaja táctica. El proyecto TACTICS, con el 
apoyo de la Agencia Europea de Defensa, se centra en el estudio y desarrollo de una SOA dedicado a redes tácticas; con el objetivo de resaltar las distintas funcionalidades de la infraestructura de seguridad hacia la aplicación eficiente de los controles de seguridad en el borde táctico (Gkioulos \& Wolthusen, 2017).

\subsubsection{SOA en el contexto administrativo}

El software administrativo está estrechamente unido a la organización interna, procesos y modelos de negocio. Este software subyace tanto en las dependencias interdepartamentales como en las relaciones exteriores de la empresa. En consecuencia, una arquitectura de software administrativo debe hacer frente a un gran número de requisitos diferentes. Muchos de estos requisitos son contradictorios, mientras que otros no están claros. En casi todos los casos, los requisitos son un blanco en movimiento por el cambio permanente de mercados, la organización de la empresa, y sus objetivos de negocio. Es por esto que el desarrollo de software administrativo se hace realmente muy complejo. Para poder brindar agilidad y eficiencia, una arquitectura de software administrativo debe contemplar características particulares, a saber: simplicidad, flexibilidad y mantenimiento, reusabilidad $\mathrm{y}$, por último, poder desacoplar la funcionalidad y la tecnología. A través de la utilización de SOA se puede ayudar a lograr los objetivos de diseño para el desarrollo de software administrativo, pues permite adaptar e integrar las aplicaciones existentes a las necesidades específicas de la organización (Montejano, Testa, García, \& Bast, 2012). Esta arquitectura más funciones en comparación con la arquitectura heredada, lo que hace que esta arquitectura sea ampliamente aceptada por la industria. (Shashwat \& Kumar , 2017).

En esta arquitectura, los clientes pueden descubrir y conectarse a los servicios disponibles a través de interfaces públicas. El lado positivo de SOA es que los clientes no conocen las plataformas, los lenguajes de programación y otros aspectos técnicos que se usan en el lado del servicio. Por lo tanto, la tarea del cliente es solo encontrar y utilizar los servicios que satisfagan sus necesidades. (Lyashov, Bereza, Babaev, Alekseenko, \& Nazvantsev, 2017)

\subsection{Beneficios para negocios de la aplicación de SOA}

Gutiérrez \& Otón (2015) puntualizan los beneficios a que se pueden acoger las empresas desde el punto de vista empresarial y tecnológico.

\subsubsection{Desde el punto de vista empresarial}

- Eficiencia. Transforma los procesos de negocio en servicios compartidos con un menor coste de mantenimiento.

- Capacidad de respuesta. Rápida adaptación y despliegue de servicios, clave para responder a las demandas de clientes, socios, administrativos y empleados.

- Adaptabilidad. Facilita la adopción de cambios añadiendo flexibilidad y reduciendo el esfuerzo.

\subsubsection{Desde el punto de vista tecnológico}

- Reduce la complejidad gracias a la compatibilidad basada en estándares frente a la integración punto a punto.

- Reutiliza los servicios compartidos que han sido desplegados previamente.

- Integra aplicaciones heredadas limitando así el coste de mantenimiento e integración.

- Beneficios en el desarrollo, ya que las aplicaciones son reutilizables, más fácil de mantener y tienen la capacidad de ampliación de las funcionalidades del sistema, exponiéndolas de una forma segura.

\subsection{Tecnologías para implementar SOA}

Según Rico \& Gómez (2007), es necesario para la implementación de un sistema sobre la arquitectura planteada por SOA, tener herramientas para la descripción y la ejecución de los servicios establecidos. Para esto, existen las tecnologías WSBPEL (Web Services - Business Process Execution Language) y WS-CDL (Web Services Choreography Description Language) para desarrollar dichas tareas y permitir la elaboración de procesos bien estructurados con la propiedad de ser una arquitectura de servicios.

WS-CDL, es un lenguaje utilizado para la definición de servicios dentro de la plataforma SOA, basado en XML y cuyo objetivo es la descripción del comportamiento de cada uno de los servicios establecidos para lograr un objetivo común. La estructura de WS-CDL es un lenguaje organizado por capas, que permiten diferentes 
niveles de expresión de las coreografías de un servicio.

WS-BPEL o especificación del lenguaje de ejecución de procesos de negocio en servicios Web, pertenece a la capa de componentes establecida en SOA y es, en conjunto con WSCDL, una alternativa para la implementación y manejo de Servicios Web.

Arquitectura WS-BPEL, se basa en un modelo de composición el cual establece varios requerimientos que se cumplen para su correcto comportamiento; el modelo de composición requiere una integración flexible para que se puedan expresar de manera adecuada los escenarios de negocios y se adapten fácilmente, requiere una composición recursiva permitiendo la integración de servicios Web e incrementando la escalabilidad y la reusabilidad, requiere separación y habilidad de composición definiendo cómo el servicio Web hace parte de un Framework pero desacoplándolo de mecanismos pertenecientes al Framework como son la calidad del servicio, requiere conversaciones estables y manejo de ciclo de vida donde el flujo de trabajo tiene definido un modelo de ciclo de vida y los servicios Web pueden mantener varias conversaciones con los servicios que interactúan con él.

\section{CONCLUSIONES}

SOA permite la integración de servicios en forma de red para que puedan ser accedidos desde diferentes aplicaciones eliminando la redundancia de datos, además posibilita el fácil acoplamiento de nuevos sistemas y la reutilización los existentes.

La aplicación de SOA en las organizaciones trae muchos beneficios, pues le permiten responder de forma efectiva y ágil ante los cambios que se presentan día a día optimizando los procesos de negocios para estar a la vanguardia en este mundo cada vez más competitivo

El internet de las cosas ha permitido la interconexión digital de aspectos de la vida cotidiana con el internet, dicha interconexión crece día a día a través de los avances tecnológicos y la innovación, para escalonar apropiadamente el internet de las cosas se requiere una arquitectura que las integre y los convierta en un gran éxito, SOA asegura la interoperabilidad entre ellos permitiendo a su vez flexibilidad y agilidad.

Universidad de Pamplona

\section{REFERENCIAS}

Chanda , J., Sengupta , S., Kanjilal , A., \& Bhattacharya , S. (2013). Behavioral and Structural Evolution of SOA from OO: An Integrated Approach. ACM SIGSOFT Software Engineering Notes, 38(5), 1-9.

Cruz, D., \& Correal, D. (2011). Estrategia dirigida por modelo para el gobierno SOA. Revista Avances en Sistemas e Informática, 8(2), 8195. Recuperado el 2017

Dutta , A., Arora , M., \& Devi, S. (2017). Census Web Service Architecture for e-Governance Applications . ICEGOV.

Escobar, D., Aguilera, A., \& Parra, Y. (2016). Propuesta metodológica para la orientación de aplicaciones informáticas hacia BPM y. Revista Cubana de Ciencias Informáticas, 3954.

García, M., Herrasti , N., Jouvray, C., \& Armentia, A. (2017). Flexible and timely on-line integration of medical services using iLand middleware. SIGBED Review, 14(2), 53-59.

Gkioulos, V., \& Wolthusen, S. (2017). Security infrastructure for service oriented architectures at the tactical edge. Advances in Intelligent Systems and Computing, 310-322.

Gutiérrez , I., \& Otón, S. (2015). Service Oriented Architecture . Obtenido de http://ceurws.org/Vol-132/paper09.pdf

Joachim, N., Beimborn, D., \& Weitzel, T. (2012). The influence of SOA governance mechanisms on IT flexibility and service reuse. The Journal of Strategic Information Systems, 22, 86-101.

Ledgard, J., \& Espinoza, A. (2010). Conceptos fundamentales de Ingeniería dirigida. Revista de Investigación de Sistemas e Informática, 9-19.

Malik, Z., Medjahed, B., \& Rezgui, A. (2016). SCARE: Reputation Estimation for Uncertain Web Services. Internet Technology.

Pereira, O., Nogueira, R., \& Aparecida, A. (2016). Integração De Sistemas De Informação Em Saúde Com A Utilização De. Journal of Information Systems and Technology Management, 255-274.

Raza, U., Lomax, J., Ghafir, I., Kharel, R., \& Whiteside, B. (2017). An IoT and business processes based approach for the monitoring and control of high value-added manufacturing processes. ICFNDS.

Sánchez, E., Clemente , P., Prieto , J., \& Rodríguez , R. (2017). MigraSOA: Migration of Legacy Web Applications to Service Oriented Architectures (SOA). IEEE LATIN 
AMERICA TRANSACTIONS, 15(7), 13061311.

Santos, L., Rico, D., \& Rincón, A. (2009). Servicios Web En Telefonía Celular Web Services in Cellular Telephony. Scientia et Technica .

Serman, D. (2010). Orientação a projetos: uma proposta de desenvolvimento de uma arquitetura orientada a serviços. JISTEM: Journal of Information Systems and Technology Management, 7(3), 619-638. Recuperado el 2017

SHENG, Q., \& RANJAN, R. (2017). A Taxonomy and Survey of Cloud Resource Orchestration Techniques. ACM Computing Surveys, 50(2).

Sordi, J., Marinho, B., \& Nagy, M. (2006). Benefícios da arquitetura de software orientada a serviços para as empresas: análise da experiência. JISTEM: Journal of Information Systems and Technology Management, 19-34.

Tarouco, H., \& Reis, A. (2010). Governança de tecnologia da informação: um panorama da adoção de modelos de melhores práticas por empresas brasileiras usuárias. Revista de Administração, Vol. 46, No. 1, 7-18.

Cristhian M Durán Acevedo, Ali Iturriago (2012). Automatización de un sistema de suministro de agua potable a través de la tecnología ZigBee. Revista Colombiana de Tecnologías de Avanzada ISSN: 16927257 - Volumen 2 - Número 20.

Parra, C., \& Herrera, J. (2013). Aplicación de los sistemas de detección de intrusos y la tecnología de agentes en el monitoreo inteligente de redes de datos. Revista Colombiana de Tecnologías de Avanzada, 106-110 ISSN: 1692-7257 - Volumen 2 Número 22.

Luz M Santos Jaimes, Anderson S Flórez Fuentes (2012). Metodología para el análisis forense en Linux. Revista Colombiana de Tecnologías de Avanzada ISSN: 1692-7257 Volumen 2 - Número 20.

Jaime Guzman-Luna, Luis Alfonso Lezcano Rodríguez, Sebastián A Gómez Arias (2013). Guzman-Luna, J., Lezcano Rodriguez, L. A. \& Gómez Arias, S. A. (2013). Caracterización de los Elementos del Diagrama de Objetivos de KAOS a partir de Lenguaje Natural. Revista Colombiana de Tecnologías de Avanzada ISSN: 1692-7257 Volumen 1 - Número 21.
Luis Fernando Gélvez R (2012). Aplicación de redes neuronales morfológicas al reconocimiento de vocablos simples. Revista Colombiana de Tecnologías de Avanzada ISSN: 1692-7257 - Volumen 1 - Número 19.

De Jesús, J. (2013). Las TICs y la Gestión empresarial.

http://www.eoi.es/blogs/mtelcon/2013/02/06/1 as-tics-y-la-gestion-empresarial/

(Consultado: 10 de octubre 2017)

Bazán , P., Fernández, A., Molinari, L., Pérez, J., \& Banchoff, M. (2017). Aplicaciones, servicios y procesos distribuidos una visión para la contrucción de software. Buenos Aires: Editorial de la Universidad Nacional de La Plata (EDULP). http://sedici.unlp.edu.ar/bitstream/hand le/10915/62354/Documento_completo. pdf-PDFA. pdf?sequence $=1$

(Consultado: 10 de octubre 2017)

Nagarajan , S. (Marzo de 2013). La Era del Almacenamiento de Datos Ágil y Siempre Disponible. computing now. Recuperado de https://sibdigital.ufpso.edu.co:2209/web/com putingnow/archive/march2013-spanish

(Consultado: 26 de octubre 2017)

GARCIA, G. (2014). "Generalidades Del Business Process". Obtenido de http://repositorio.unapiquitos.edu.pe/bitstrea m/handle/UNAP/4512/German_Tesis_Titulo _2014.pdf?sequence $=1$

(Consultado: 8 de Septiembre 2017)

CAR. (2008). Arquitectura Orientada a Servicios (SOA) Cómo reformular la Arquitectura Corporativa.

http://www.kybele.etsii.urjc.es/docencia/IS_L $\mathrm{ADE} / 2012$ -

2013/Material/CAR\%20Accenture\%20$\% 20$ SOA\%5B1\%5D.pdf

(Consultado: 15 de Septiembre 2017)

Oviedo, J. (2016). Diseño arquitectónico y mecanismos de gobierno para SOA. Computing Now. Obtenido de https://sibdigital.ufpso.edu.co:2209/web/chap ters/Jaime-Oviedo

Gonzáles, R. (2010). Implementación de una api de información bancaria para la sbif. Obtenido de http://repositorio.uchile.cl/bitstream/handle/2 250/104108/cfgonzalez_ro.pdf? sequence $=3$ \&isAllowed $=y$

(Consultado: 12 de octubre 2017)

Alfonso \& Enciso (2010). Diseño de un sistema de notificación de transacciones de recaudos entre entidades financieras y empresas 
facturadoras, utilizando una arquitectura orientada a servicios (SOA). Recuperado de https://repository.javeriana.edu.co/bitstream/h andle/10554/7529/tesis356.pdf? sequence $=1 \&$ isAllowed $=\mathrm{y}$

(Consultado: 11 de octubre 2017)

IEEE Computer Society. (2017). La Fundación de Arquitectura de Referencia para la Arquitectura Orientada a Servicios Versión 1.0. Obtenido de https://standards.ieee.org/develop/proj ect/1723.2.html (Consultado: 05 de Septiembre 2017)

Granados. (2014). Diseño de una arquitectura soa aplicada en un sistema de consultorio médico virtual.

Recuperado de http://www.ptolomeo.unam.mx:8080/x mlui/bitstream/handle/132.248.52.100/ 4067/Eduardo\%20Tesis\%20SOA\%20SCM V.pdf?sequence $=1$

(Consultado: 27 de agosto 2017)

Gonzáles , M. (2011). ESTUDIO DE ARQUITECTURAS DE. Recuperado de http://upcommons.upc.edu/bitstream/h andle/2099.1/12312/ESTUDIO_DE_ARQ UITECTURAS_DE_REDES_ORIENTADAS_A SERVICIO.pdf?sequence $=1$

(Consultado: 07 de Octubre 2017)

INMETRICS. (2017). La gobernanza de TI le asegura una ventaja competitiva a la empresa. Obtenido de http://www.inmetrics.com.br/es/governancade-ti-garante-vantagem-competitiva-paraempresa/

(Consultado: 10 de Septiembre 2017)

Londoño, J. (2014). Modelo funcional de Integración de la Arquitectura Empresarial de ' $\mathrm{N}$ ' entidades alrededor de un grupo empresarial. Un enfoque de orientación a servicios y modelado de capacidades de negocio. Obtenido de http://www.bdigital.unal.edu.co/46046/1/703 22207.2014.pdf

(Consultado: 25 de Octubre 2017)

Cardona, V. (2014). BplSoa: Framework para el desarrollo de líneas de procesos de negocios orientadas a servicios. Obtenido de http://www.bdigital.unal.edu.co/45442/

1/79988120.2014.pdf (Consultado: 25 de Octubre 2017)

Bazán , P. (2009). Un modelo de integrabilidad con SOA y BPM. Obtenido de http://postgrado.info.unlp.edu.ar/Carreras/Ma
gisters/Redes_de_Datos/Tesis/Bazan_Patricia .pdf

(Consultado: 03 de Octubre 2017)

Arango, M., Londoño, J. E., \& Zapata, J. (2010). Arquitectura orientada a servicios en el contexto de la arquitectura empresarial. Obtenido http://www.bdigital.unal.edu.co/28772/1/266 00-93259-1-PB.pdf

(Consultado: 19 de octubre 2017)

Shashwat, A., \& Kumar , D. (2017). A Service Identification Model For Service Oriented Architecture. Obtenido de http://ieeexplore.ieee.org/document/7977299/

(Consultado: 27 de octubre 2017)

El Economista America. (2007). Nortel e IBM utilizan la arquitectura SOA para conseguir más eficiencia en las comunicaciones entre clientes, empleados y colaboradores. Obtenido de http://www.eleconomistaamerica.com/empres as-finanzas/noticias/315368/10/17/Nortel-eIBM-utilizan-la-arquitectura-SOA-paraconseguir-mas-eficiencia-en-lascomunicaciones-entre-clientes-empleados-ycolaboradores.html

(Consultado: 15 de octubre 2017)

Lyashov, M., Bereza, A., Babaev, A., Alekseenko, J., \& Nazvantsev, D. (2017). The Technique of Creating Distributed Computing Systems based on Service-Oriented Architecture. Obtenido http://ieeexplore.ieee.org/document/7991710/

(Consultado: 26 de octubre 2017)

Spiess, P., Karnouskos, S., Guinard, D., Savio, D., Baecker, O., Moreira, L., \& Trifa, V. (2016). SOA-based Integration of the Internet of Things in Enterprise Services. Recuperado, de

http://citeseerx.ist.psu.edu/viewdoc/download ?doi=10.1.1.208.634\&rep=rep1\&type $=$ pdf

(Consultado: 19 de octubre 2017)

Montejano, G., Testa, O., García, P., \& Bast, S. (2012). Generación de Sistemas de Software: metodología de desarrollo basada en SOA . Obtenido de http://sedici.unlp.edu.ar/bitstream/handle/109 15/19046/Documento_completo.pdf?sequenc $\mathrm{e}=1$

(Consultado: 15 de octubre 2017)

Fortino, G., Ganzha, M., Palau, C., \& Paprzycki, M. (2016). Interoperabilidad para la Internet de las Cosas. Computing Now.

Carvajal, L. (2014). Plan De Negocio Para Empresa De Servicios Informáticos 
Ibáñez, M. (2015). Implementación de un framework para la programación de. Obtenido de http://repositorio.uchile.cl/bitstream/handle/2 250/133867/Implementacion-de-unframework-para-laprogramacion.pdf?sequence $=1 \&$ is Allowed $=\mathrm{y}$

(Consultado: 20 de octubre 2017)

ETCHART, I. (2015). Diseño De Una Estrategia Para Incorporar Un Sistema De Historia. Obtenido de http://repositorio.uchile.cl/bitstream/handle/2 250/133318/Diseno-de-una-estrategia-paraincorporar-un-sistema-dehistoria.pdf? sequence $=1 \&$ is Allowed $=\mathrm{y}$

(Consultado: 23 de octubre 2017)

Juneca, G., Dournaee, B., Natoli, J., \& Birkel, S. (2008). Mejora del rendimiento de los sistemas sanitarios con arquitectura orientada a servicios.

Montejano, G., Testa, O., García, P., \& Bast, S. (2012). Generación de Sistemas de Software: metodología de desarrollo basada en SOA.

Hernández , Y., Leal , N., Nieto , W., Jimeno , M., \& Muñoz , H. (2017). Framework for the Development of Business Intelligence Using Computational Intelligence and ServiceOriented Architecture.

Zavvar, M., Garavand, S., \& Sabbagh, E. (2017). Measuring Service Quality in ServiceOriented Architectures Using a Hybrid Particle Swarm Optimization Algorithm and Artificial Neural Network (PSO-ANN).

Rico, J., \& Gómez, J. (2007). Documento de estado del arte en SOA y Cálculo de PI.

Mohamad, A. (Mayo de 2007). SOA Governance: Framework

Romero, C. (2012). Modelado e implementación de un proceso de negocio BPM mediante herramientas SOA. Obtenido de https://repository.javeriana.edu.co/bitstream/h andle/10554/7546/tesis649.pdf? sequence $=1 \&$ isAllowed $=\mathrm{y}$ 